\title{
Finite Difference Numerical Method: Applications in Lightning Electromagnetic Pulse and Heat Diffusion
}

\author{
*Olufunke G. Darley, Adetokunbo A. Adenowo and Abayomi I. Yussuff \\ Department of Electronic \& Computer Engineering, Lagos State University, Epe Campus, Nigeria \\ \{funke_darleyladetokyom\}@yahoo.com labayomi.yussuff@lasu.edu.ng
}

Received: 12-MAR-2021; Reviewed: 06-APR-2021; Accepted: 28-JUL-2021

http://dx.doi.org/10.46792/fuoyejet.v6i3.621

\begin{abstract}
The finite difference time domain (FDTD) is a technique of the finite difference numerical method and is a simple but powerful and versatile tool that has been widely applied in many scientific and engineering problems. A typical application of the technique is in dealing with electromagnetic (EM) wave interactions with physical structures. This technique has been used to solve governing equations of various systems through obtaining numerical approximations to the time-dependent differential equations for computer simulations. This paper demonstrates the accuracy and versatility of the application of FDTD method by applying it to examine the effect of lightning electromagnetic pulse (LEMP) on a transmission line using a cross-linked polyethylene (XLPE) insulated power cable, as well as to analyze heat diffusion in a microchip heat sink made from Aluminium Alloy 6061. The effect of LEMP on a transmission line showed that the higher the values of the line parameters, the larger the voltages that will be induced on the line and that bigger values of finite difference (FD) parameters give a more accurate model subject to a stability criterion. Accurate modelling of induced voltages ensures that appropriate mitigating techniques can be deployed to reduce or eliminate the damaging effect of these on electrical and/or electronic devices/systems. Similarly, proper modeling of a heat sink provides the ability to closely estimate heat diffusion at product design stage such that a design is confirmed as workable before manufacture; thereby saving cost.
\end{abstract}

Keywords- Finite Difference, Time Domain, Engineering Applications, Heat Diffusion, Lightning Electromagnetic Pulse

\section{INTRODUCTION}

A mathematical model describes a system using mathematical concepts and language; it aims to facilitate proper explanation of a system or to study the effects of different components, as well as to make predictions on patterns of behaviour. Modelling enables the study of a physical system without incurring the expenditure associated with experiments or constructions and sometimes lack of accessibility to the system under investigation. Mathematical modelling involves representing the system of interest in the form of ordinary differential equations (ODEs) or partial differential equations (PDEs) as the case may be with various methods of solving differential equations (DEs) applied to the model (See Fig. 1). While an exact or analytical (closed form) solution is desired, this is often times, not possible either because an exact solution does not exist and even when it does, the computational complexity and the time expended in getting the solution makes it unattractive. Numerical methods provide alternative approximate solutions within acceptable margins of error, with various methods available, close approximations to desired accuracy attainable and fast computations within acceptable time limit possible. Whatever method is used, it should have consistency, stability and convergence. For many cases in the natural sciences and engineering, mathematical models used are based on ODEs and PDEs with the latter being more common.

\footnotetext{
${ }^{*}$ Corresponding Author
}

Section B- ELECTRICAL/COMPUTER ENGINEERING \& COMPUTING SCIENCES Can be cited as:

Darley O., Adenowo A. and Yussuff A. (2021): Finite Difference Numerical Method: Applications in Lightning Electromagnetic Pulse and Heat Diffusion, FUOYE Journal of Engineering and Technology (FUOYEJET), 6(3), 27-33. http://dx.doi.org/10.46792/fuoyejet.v6i3.621
The Finite Difference Method (FDM) is the most direct and dominant approach to discretizing PDEs and is suited for and is efficient in solving problems of regular grid and box-like geometries (Sjodin, B., 2016). It can be used to solve all types of DEs in a bounded domain including linear and non-linear, time-dependent and time-independent problems (Zhou, 1993; Robertsson and Blanch, 2014; Kumar, 2014; Tsegaye and Purnachandra, 2016; Feizi et al, 2018; Chackraverty et al, 2019; Onishi et al, 2019; Wai and Tint, 2019). The FDM has two sources of error; round off error and truncation/discretization error. (Fauset, 1999; Kumar, 2014).

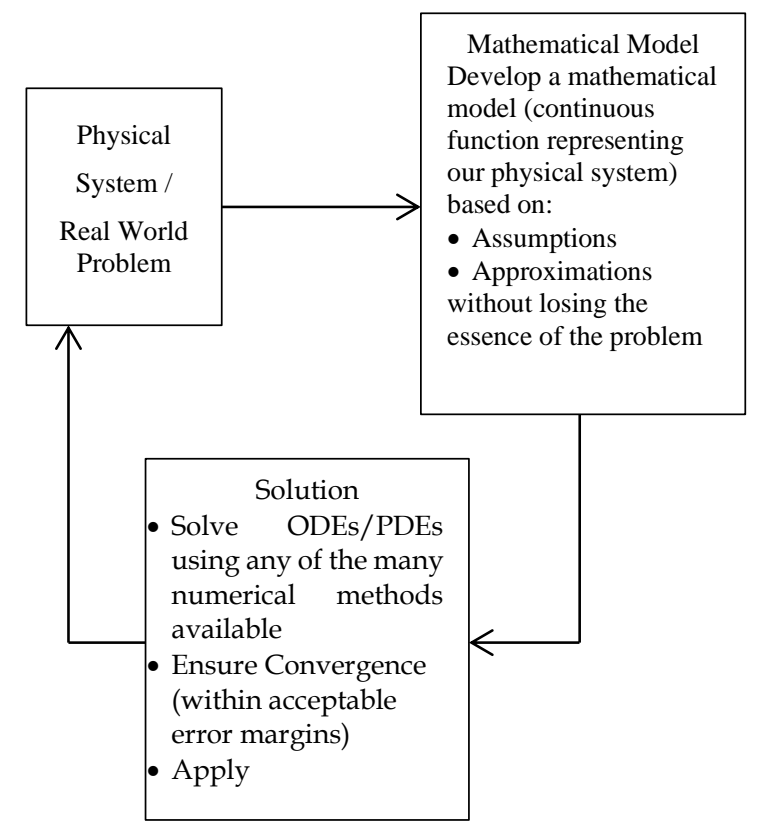

Fig. 1: Block Diagram representing the inter-relationship between a physical system, its model and numerical solution

The finite-difference time-domain (FDTD), an explicit time domain method, is very popular and has been widely studied and applied in many scientific and engineering problems dealing with electromagnetic (EM) 
wave interactions with material structures (Smy et al, 2018). It is a versatile tool for electrical engineers and is a simple and powerful method to solve Maxwell equations by numerical simulation of EM wave propagation. To solve an EM problem, the idea is to simply discretize, both in time and space, the Maxwell's equations with central difference approximations resulting in a large set of linear algebraic equations which are written in matrix form (1) and then solved. The originality of the aforementioned idea (Yee, 1966) lies in the allocation in space of the electric and magnetic field components, and the marching in time for the procedure to evolve.

$$
A X=B
$$

where

A represents the matrix of known coefficients

$X$ represents the matrix (column vector) of unknown variable

$B$ represents the matrix of known constants

In this paper, the Explicit FDTD method is applied to analyse the effect of voltage induced on a transmission line due to lightning strike (LEMP) using the wave equation otherwise known as the Telegrapher's Equation (Pozar, 2005). A one-dimensional solution to the equation is presented. Using MATLAB, the effects of changes in transmission line parameters (inductance and capacitance) and explicit FDTD parameters (number of space and time steps) were presented. The FDTD method was also applied to analyse heat diffusion (Sam R, 2020) in a heat sink and a two-dimensional solution presented; using MATLAB. With the illustration of ease of application of FDM/FDTD methods, it is expected that more studies on its application to fields outside engineering will be carried out.

\section{Application OF FDTD to LEMP ON A TRANSMISSION LINE}

\subsection{Propagation Of lemp in Free Space and IMPINGEMENT ON A TRANSMISSION LINE}

When lightning strikes a target, LEMP is produced and EM waves are propagated in free space, to nearby materials, structures and devices/equipment via oscillating electric and magnetic fields that are at right angles to each other. LEMP can impinge on a transmission line directly or indirectly by reflection from the ground, if lightning strikes the ground. Overhead structures such as transmission lines (telecommunications and power lines) can receive energy from both direct and reflected pulses. These pulses have a steep leading edge that quickly reaches its maximum value and thereafter trailed by a sequence of pulses of decreasing energy. Thus, LEMP is a transient disturbance having a short burst of EM energy spread over a range of frequencies. It can be modelled by a double-exponential curve (Figure 2) which climbs steeply, quickly reaches a peak and then decays more slowly. LEMP usually induces a corresponding signal in the surrounding environment or material which can be damaging to the aforesaid; thus, necessitating the need to analyse its effect on these structures such that mitigating techniques can be put in place.

Coupling can occur in four different ways through capacitive (electric) and inductive (magnetic) coupling between electrical conductors, EM radiation and electric conduction depending on the source of energy. (Laughton and Warne, 2003; Pejtamalli and Cipo, 2015). Coupling can be represented by a characteristic damped sine wave shown as a high frequency sine wave growing and decaying within the longer-lived envelope of the double-exponential curve (Fig. 3). A damped sine wave normally has much lower energy and a narrower frequency spread than the original pulse, due to the transfer characteristic of the coupling mode (Pejtamalli and Cipo, 2015).

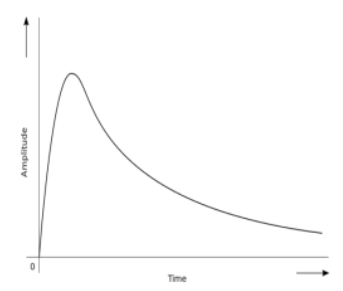

Fig. 2: Diagram of EMP- Double Exponential (Wikimedia, 2013)

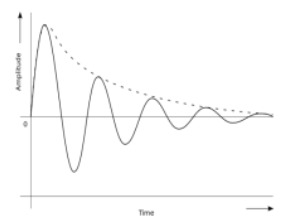

Fig.3: Diagram EMP- Damped Sinewave (Wikimedia, 2013)

At far enough distances from the point of lightning strike (50 km in this case), the EM field generated is dominated by its radiation component; and therefore, can be represented by a plane wave. Other modes of transmission will be dominant at shorter distances.

In line with Pozar (2005), a transmission line can be represented by a lumped-element equivalent circuit with distributed parameter from which the wave equation can be derived as below:

$$
\frac{\partial^{2} v(z, t)}{\partial t^{2}}+\left(\frac{L G+R C}{L C}\right) \frac{\partial v(z, t)}{\partial t}+\left(\frac{R G}{L C}\right) v(z, t)=\left(\frac{1}{L C}\right) \frac{\partial^{2} v(z, t)}{\partial z^{2}}
$$

where,

$\mathrm{R}$ represents series resistance per unit length for the two conductors $(\Omega / \mathrm{m})$

$\mathrm{L}$ represents series inductance per unit length for the two conductors $(\mathrm{H} / \mathrm{m})$

$\mathrm{G}$ represents shunt conductance for each unit length $(\mathrm{S} / \mathrm{m})$ $C$ represents shunt capacitance for each unit length $(\mathrm{F} / \mathrm{m})$ $\mathrm{v}$ represents induced voltage $(\mathrm{V})$

In practice, losses (such as skin effect, heating, coupling, radiation) will occur along the transmission line. For simplicity, a lossless line is assumed by equating $R$ and $G$ in (2) to zero. Thus (2) becomes

$$
\frac{\partial^{2} v(z, t)}{\partial t^{2}}-k^{2} \frac{\partial^{2} v(z, t)}{\partial z^{2}}=0
$$

where $k=1 / \sqrt{L C}$ is the wave constant.

The wave equation is classified as a hyperbolic PDE and the explicit method of FDM for time-dependent DEs was used. The central differencing scheme was applied to both space and time. Thus (3) can be rewritten and represented 
by finite difference equations (Smith, 1985; Pozar 2005; Kumar, 2014; Chakraverty et al., 2019) as follows:

$$
\begin{gathered}
\frac{v_{i, j+1}-2 v_{i, j}+v_{i, j-1}}{(\Delta t)^{2}}=k^{2} \frac{\left(v_{i+1, j}-2 v_{i, j}+v_{i-1, j}\right)}{(\Delta z)^{2}} \\
v_{i, j+1}-2 v_{i, j}+v_{i, j-1}=\left(\frac{k \Delta t}{\Delta z}\right)^{2}\left(v_{i+1, j}-2 v_{i, j}+v_{i-1, j}\right) \\
v_{i, j+1}-2 v_{i, j}+v_{i, j-1}=r^{2}\left(v_{i+1, j}-2 v_{i, j}+v_{i-1, j}\right)
\end{gathered}
$$

where $r=\frac{k \Delta t}{\Delta z}$

$$
v_{i, j+1}=r^{2} v_{i+1, j}+2\left(1-r^{2}\right) v_{i, j}+r^{2} v_{i-1, j}-v_{i, j-1}
$$

Initial Conditions are given as:

$$
\begin{aligned}
& v(z, 0)=f_{1}(z)=0, v(z, T)=f_{2}(z)=\operatorname{Sin}(\pi z) \\
& \text { for } 0 \leq z \leq L
\end{aligned}
$$

Boundary Conditions are given as: $v(0, t)=g_{1}(t)=0, v(L, t)=g_{2}(t)=0$ for $0 \leq t$

For the solution to be stable, the following requirements must be met (stability criterion):

1. Sum of coefficients of the $v(:, j)$ terms must be less than or equal to 2 ; this is satisfied for all choices of $r$ since $r^{2}+2\left(1-r^{2}\right)+r^{2}=2$

2. No coefficient of $v(:, j)$ is negative; this requires that $\mathrm{r} \leq 1$.

\subsection{Cable Parameters}

A cross-linked polyethylene (XLPE) insulated power cable represents a transmission line and its parameters are given below:

$$
\begin{aligned}
& \mathrm{R}=6.33 \times 10^{2}(\mathrm{Ohm} / \mathrm{m}) ; \mathrm{L}=1.87 \times 10^{-7}(\mathrm{H} / \mathrm{m}) \\
& \mathrm{G}=6.69 \times 10^{-15}(\mathrm{~S} / \mathrm{m}) ; \mathrm{C}=1.42 \times 10^{-10}(\mathrm{~F} / \mathrm{m})
\end{aligned}
$$

The parameters of the FDTD equations (numbers of space and time steps, wave constant) are varied to obtain the effects of LEMP on the transmission line. Two cases considered are dependent on distance of transmission line from lightning strike:

i) For distances $\leq 50 \mathrm{~km}$, induced voltage is represented by a damped sine (transient) wave given as:

$$
v(z, t)=A e^{-z(i)} \operatorname{Sin}(\pi(z(i)) \quad(10)
$$

ii) For distances $>50 \mathrm{~km}$, induced voltage is represented by a uniform sine (plane) wave given as:

$$
v(z, t)=\operatorname{Sin}(\pi(z(i))
$$

\section{Application of FDTD to Heat Diffusion in a HEAT SINK}

\subsection{Heat TRANSFER In a HEat Sink}

The underlying principle governing a heat sink is that thermal energy will be transferred from a higher temperature device to a lower temperature fluid medium; as shown by the heat equation (Hancock, 2006; Kreith et al, 2011). The fluid medium can be air, water, refrigerants or oil. For heat sinks of electronic devices to be effective, its temperatures must be higher than that of the surroundings such that heat can be transferred through convection, radiation, and/or conduction. A heat sink can be represented by a two-dimensional diffusion equation (Selvadurai and Selvadurai, 2000; Becker and Kaus, 2014; Wai and Tint, 2019) as shown below:

$$
C \rho \frac{d u}{d t}=\frac{d}{d x}\left(K_{0 x} \frac{d u}{d x}\right)+\frac{d}{d y}\left(K_{0 y}\right)+Q
$$

where

$\mathrm{C}$ represents specific heat capacity $(\mathrm{J} / \mathrm{kg} \mathrm{K})$

Q represents density $\left(\mathrm{Kg} / \mathrm{m}^{3}\right)$

$\mathrm{K}_{0 \mathrm{x}}$ and $\mathrm{K}_{0 \mathrm{y}}$ are the thermal conductivities in $x$ and $y$ direction.

$Q$ is the radiogenic heat production (volumetric rate of internal heat generation $\left(\mathrm{W} / \mathrm{m}^{3}\right)$.

If the thermal conductivity is homogeneous and isotropic $K_{0 x}=K_{0 y}$ and (12) can be rearranged and rewritten as

$$
\frac{d u}{d t}=\alpha\left(\frac{d^{2} u}{d x^{2}}+\frac{d^{2} u}{d y^{2}}\right)
$$

where

$\alpha(K / C \rho)$ is thermal diffusivity $\left(\mathrm{m}^{2} / \mathrm{s}\right)$.

The ability to estimate heat diffusion in a heat sink through a numerical method is of particular importance in product design ensuring that a design is workable before a physical model is made; thereby making it a cost saving measure. Hence, the selection of combinations of time and space steps is important (Gerald, 2011). The heat equation is a parabolic PDE and the explicit method of FDM for time-dependent DEs is used. The forward differencing scheme is applied to time while the central differencing is applied to space (forward time central space (FTCS) method). Thus (13) can be rewritten and represented by finite difference equations as follows (Fauset, 1999, Wai and Tint, 2019):

$$
\frac{1}{\Delta t}\left[u_{i, j+1}-u_{i, j}\right]=\alpha\left[\frac{u_{i-1, j}-2 u_{i, j}+u_{i+1, j}}{\Delta x^{2}}+\frac{u_{i, j-1}-2 u_{i, j}+u_{i, j+1}}{\Delta y^{2}}\right]
$$

Assuming that the heat sink base is square, then $\Delta x=\Delta y$; and multiplying (14) by $\Delta t$ gives

$$
\begin{gathered}
{\left[u_{i, j+1}-u_{i, j}\right]=\alpha \frac{\Delta t}{\Delta x^{2}}\left[u_{i-1, j}-2 u_{i, j}+u_{i+1, j}+u_{i, j-1}-\right.} \\
\left.2 u_{i, j}+u_{i, j+1}\right]
\end{gathered}
$$

Replacing $\alpha \Delta t / \Delta x^{2}$ by parameter, $r$, and rearranging (15), it can be re-written as

$$
\begin{gathered}
u_{i, j+1}=r u_{i-1, j}+(1-4 r) u_{i, j}+r u_{i+1, j}+r u_{i, j-1}+ \\
r u_{i, j+1}
\end{gathered}
$$

Initial conditions: $u(x, 0)=f(x)$ for $0<x<a$

Boundary conditions:

$$
u(0, t)=g_{1}(t) \text { and } u(a, t)=g_{2}(t) \text { for } 0<t<T
$$

For the solution to be stable, steps taken must be such that $0<\mathrm{r} \leq 0.25 . \mathrm{m}$

\subsection{Heat Sink PaRAmeters}

A heat sink made of Aluminium Alloy 6061 (Offshore Direct Metals, 2020) is considered and its parameters are given as follows:

Thermal Conductivity, $\mathrm{K}=151-202 \mathrm{~W} / \mathrm{m}-\mathrm{C}$; Specific heat capacity, $\mathrm{C}=897 \mathrm{~J} / \mathrm{kg} \mathrm{K}$ and

Density, $\varrho=2700 \mathrm{Kg} / \mathrm{m}^{3}$. 


\section{Results ANd Discussion \\ 4.1 LEMP ON A TRANSMISSION LINE}

When a LEMP impinges on a transmission line, the induced voltage can be a damped sine wave (Figures 4 7) or a plane wave (Figures 8 - 11), depending on the distance of the transmission line from the source or point of lightning strike. It can be observed from the time response that the induced voltage is influenced by transmission line parameters; inductance, $\mathrm{L}$ and capacitance, C. Higher values of L and C, (and thus lower values of $\mathrm{k}$ ), lead to greater disturbances on the transmission line in terms of higher maximum voltages and longer transient times, i.e., the time it takes the transient voltage on the line to die out.

For the FDTD parameters, use of higher number of time and space steps, gave a more accurate representation of voltages induced on the transmission line; hence, resulting in a better model. In the quest to improve accuracy by increasing number of steps however, it is critical that the stability criterion must be met. Thus, the choice of FDTD parameters is very important in modelling. To analyse the time response of the XLPE cable (Figures 6 and 7), a more accurate representation was obtained with $\mathrm{Nt}=2500$. It is expected that its time response will be useful in designing mitigation techniques for system protection against surges. This is because induced voltages due to nearby lightning strikes can cause damage to line apparatus and connected electronic devices especially when the Surge Withstand Capability (SWC) is exceeded (Nucci et al, 2004). Through the use of numerical simulations, however, appropriate lightning and surge protection schemes can be implemented such that system quality is not compromised (Palone et al., 2009).

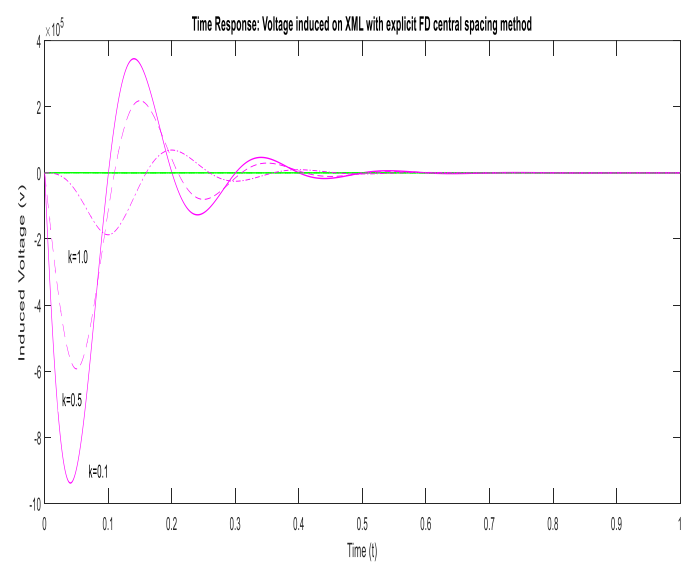

Fig. 4: Time response with $\mathrm{Nt}=2500$ with varying values of $\mathrm{k}$

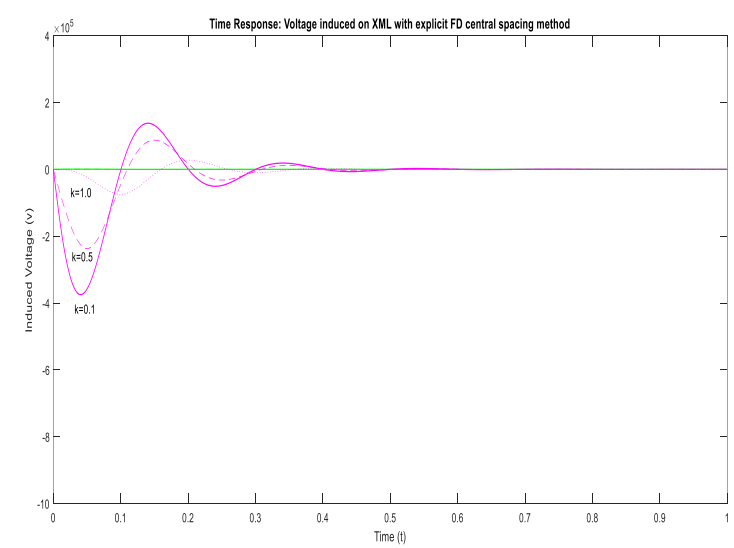

Fig. 5: Time response with $\mathrm{Nt}=1000$ with varying values of $\mathrm{k}$

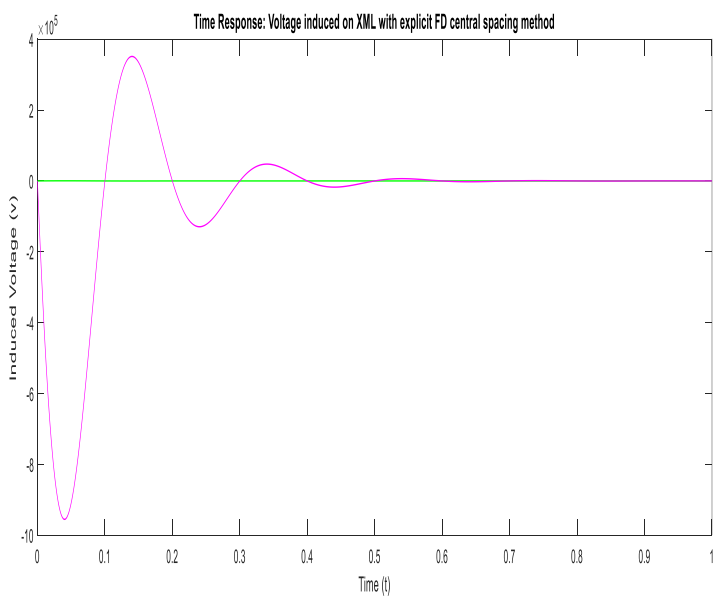

Fig. 6: Time response of XLPE Cable with $\mathrm{Nt}=2500$

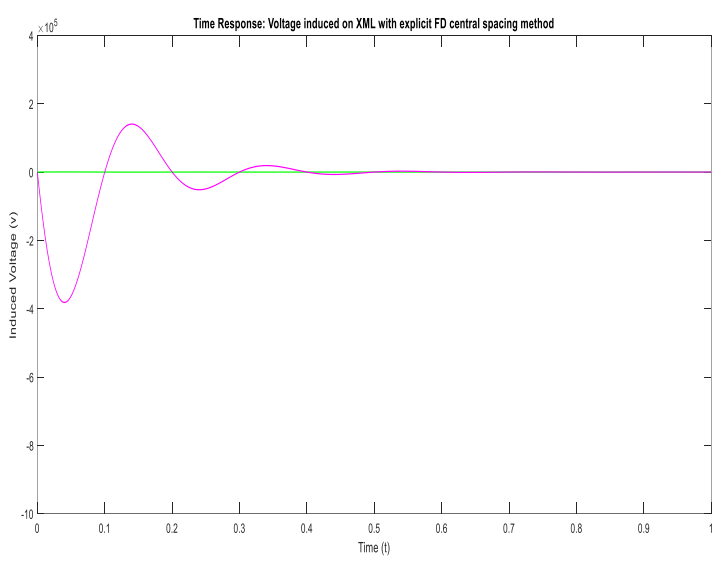

Fig. 7: Time response of XLPE Cable with $\mathrm{Nt}=1000$

For the uniform sine wave, induced voltage is propagated down the line continuously (Figures 8 - 11). Unlike the damped sine wave which is transient, the uniform sine wave may be perpetuated for long periods of time due to reflections on the line caused by impedance mismatch between the cable and its terminations. This will present as a 'ringing' effect. This feature must also be taken into consideration in designing mitigating techniques. In real life, however, transmission lines are lossy and there will be some damping of the induced voltage depending on the characteristics of the line. 


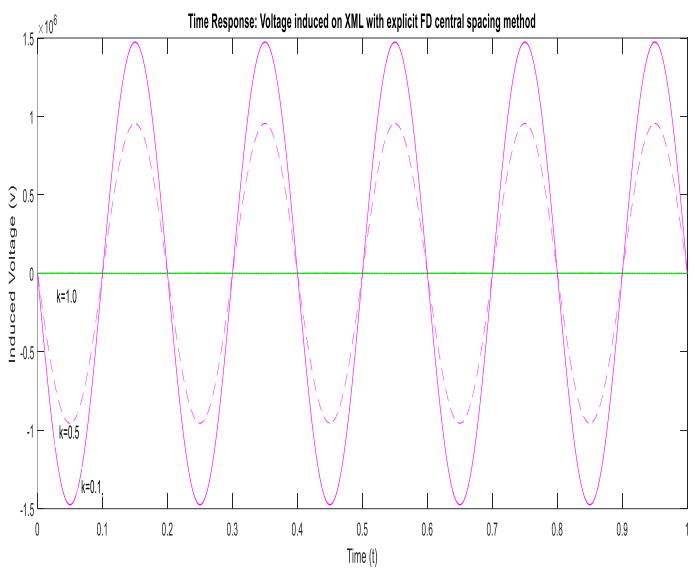

Fig. 8: Time response with $\mathrm{Nt}=2500$ with varying values of $\mathrm{k}$

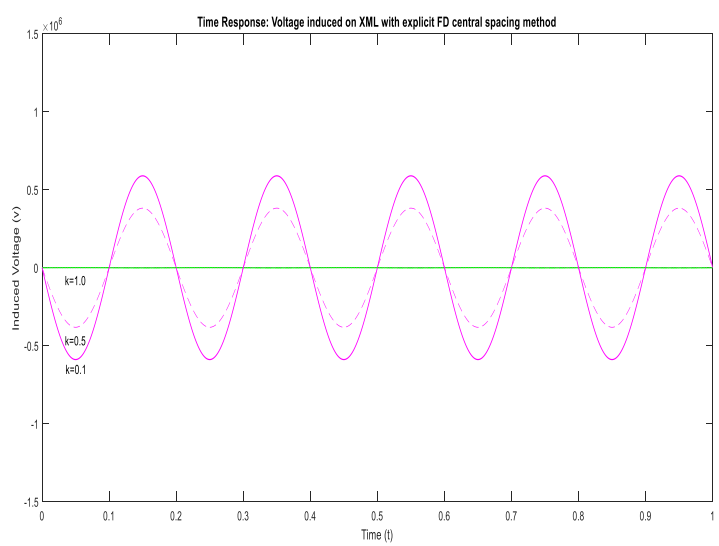

Fig. 9: Time response with $\mathrm{Nt}=1000$ with varying values of $\mathrm{k}$

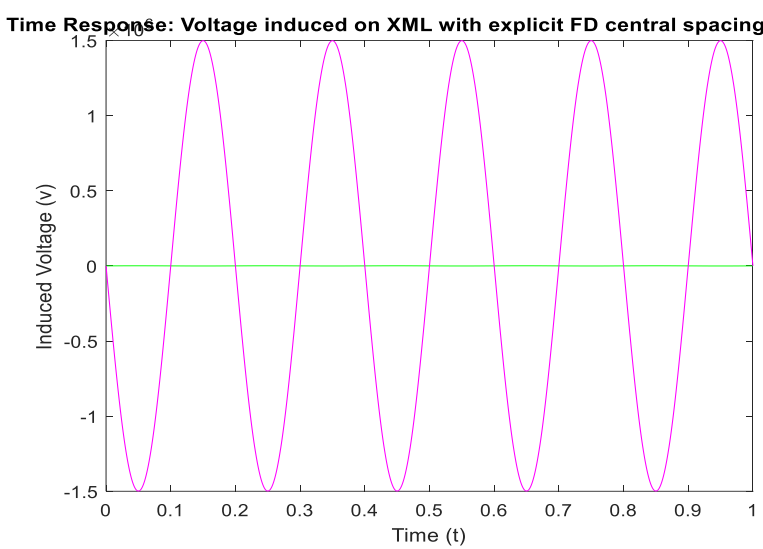

Fig. 10: Time response of XLPE Cable with $\mathrm{Nt}=\mathrm{Nz}=2500$

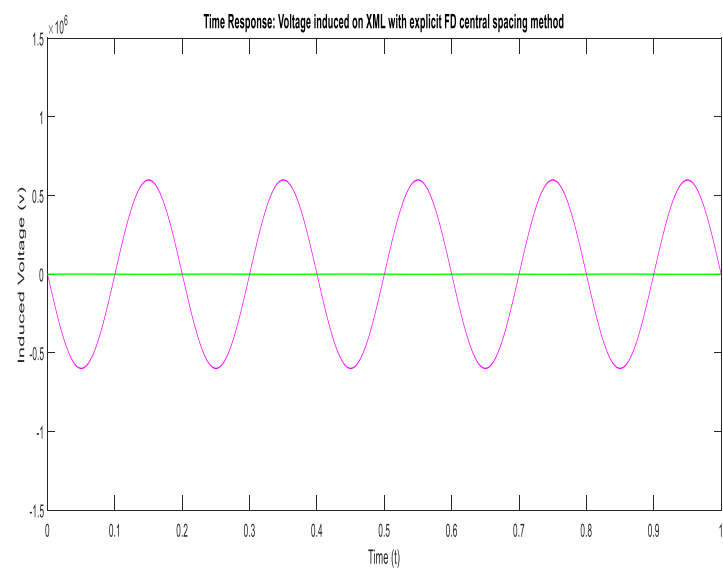

Fig. 11: Time response of $\mathrm{XLPE}$ Cable with $\mathrm{Nt}=\mathrm{Nz}=1000$

\subsection{Heat diffusion in a Heat Sink}

Selection of appropriate values of time, $t$, number of time steps, nt and number of space steps, $n x=n y$, determines the accuracy of the model in showing heat diffusion or temperature distribution in a heat sink over varying time period. (Figures 12 - 14).

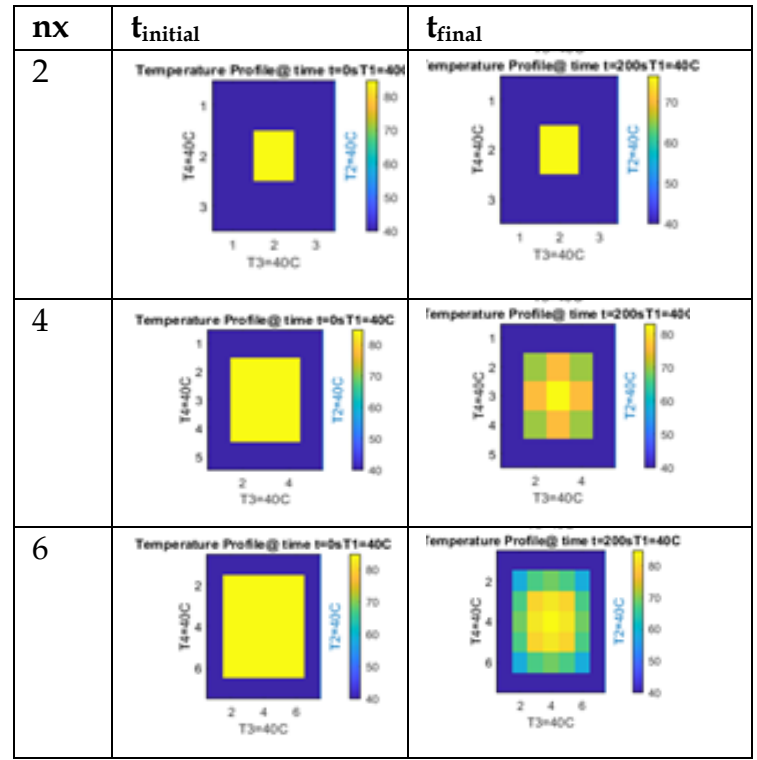

Fig. 12a: Temperature profile showing the effect of varying $\mathrm{nx}$ $(\mathrm{t}=200, \mathrm{nt}=2, \mathrm{nx}=2,4,6)$.

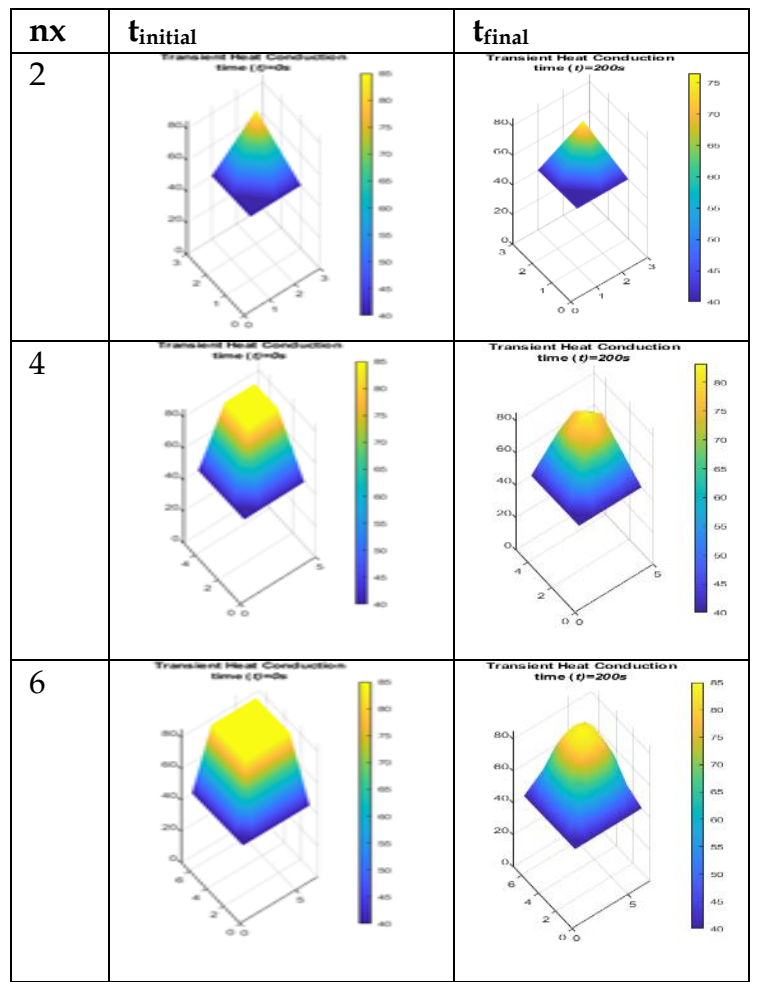

Fig. 12b: Transient heat sink behaviour with varying $n x(t=200$ $\mathrm{nt}=2, \mathrm{nx}=2,4,6)$

Figures $12 a \& b ; 13 a \& b$, show the effect of number of space steps and time steps, respectively, on simulation. With increase in number of steps, the model gives a better illustration of heat diffusion in the heat sink. 


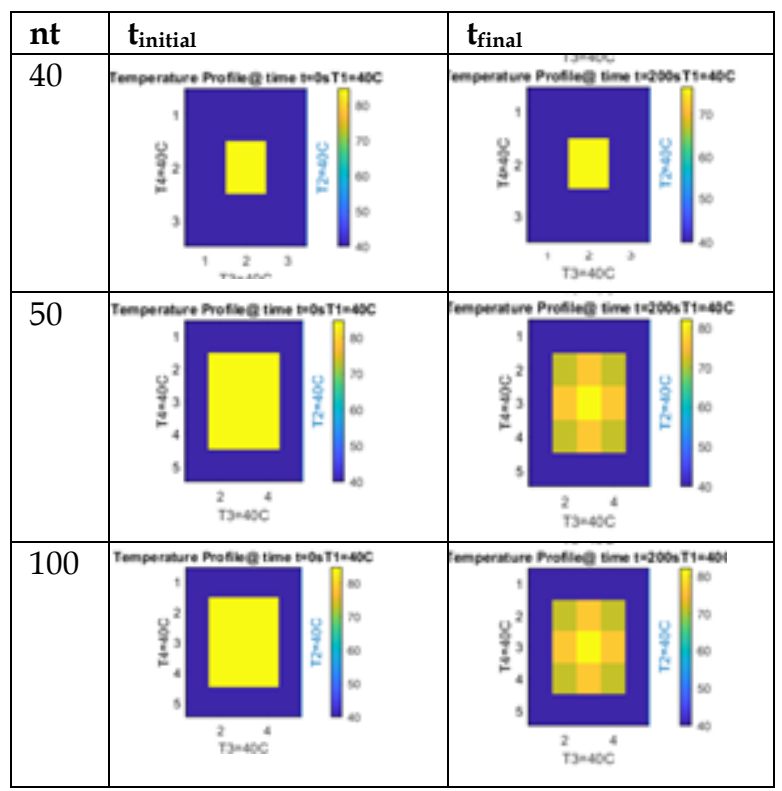

Fig. 13a: Temperature profile showing the effect of varying $\mathrm{nt}(\mathrm{t}=200$, $\mathrm{nx}=4, \mathrm{nt}=40,50,100$ )

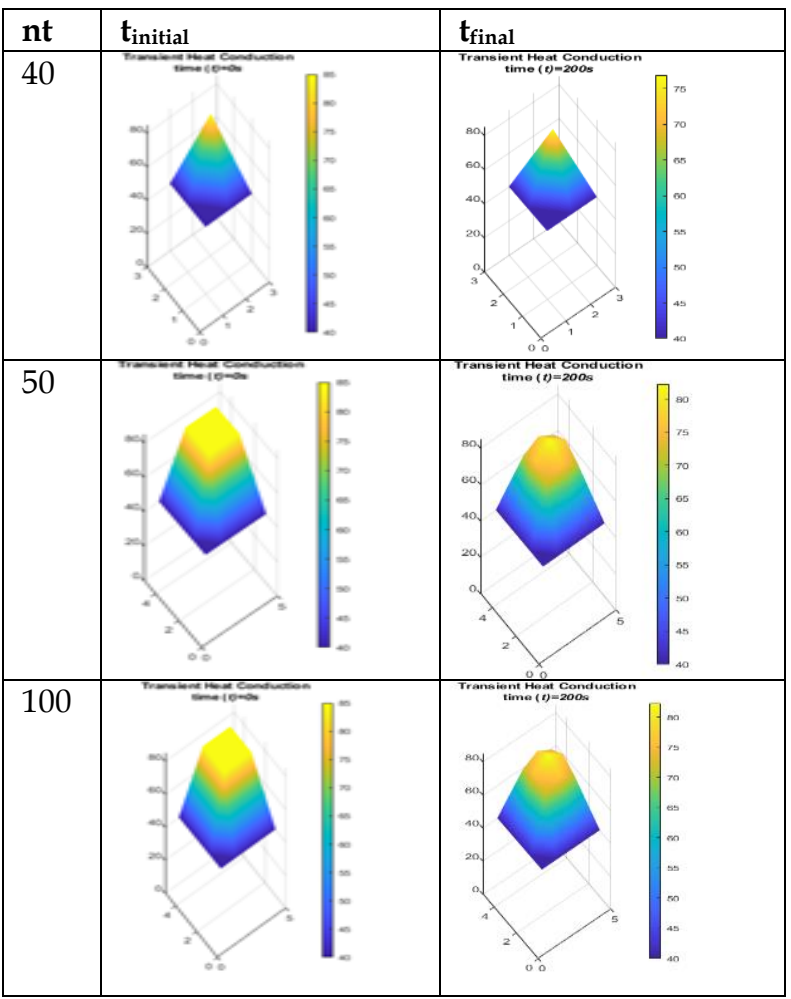

Fig. 13b: Transient heat behaviour with varying $\mathrm{nt}(\mathrm{t}=200, \mathrm{nx}=4$, $\mathrm{nt}=40,50,100$ )

By using appropriate FDTD parameters, the process of heat diffusion from the interior of the heat sink (at $85^{\circ} \mathrm{C}$ ) to its boundaries (at $40^{\circ} \mathrm{C}$ ) can be clearly observed. Figures $14 \mathrm{a} \& \mathrm{~b}$ show diffusion of heat from temperature of $85^{\circ} \mathrm{C}$ to $61^{\circ} \mathrm{C}, 51^{\circ} \mathrm{C}, 49^{\circ} \mathrm{C}$ and lastly $46.2^{\circ} \mathrm{C}$ which is close to the boundary temperature of $40^{\circ} \mathrm{C}$.

Heat diffusion in a heat sink is a gradual process and can be better observed for longer time, $t$. The longer the time under consideration, the cooler the interior of the heat sink becomes. Notably, there is an optimal value of time when transfer of heat from the highest temperature point to other parts of the heat sink has been completed. Any further increase in time is of no consequence. Also, care must be taken in choosing FDTD parameters such that the solution does not become unstable (stability criterion must be met). With the right choice, an accurate model will be obtained and the selection of these parameters would have ensured physically meaningful approximate solution for the diffusion equation.

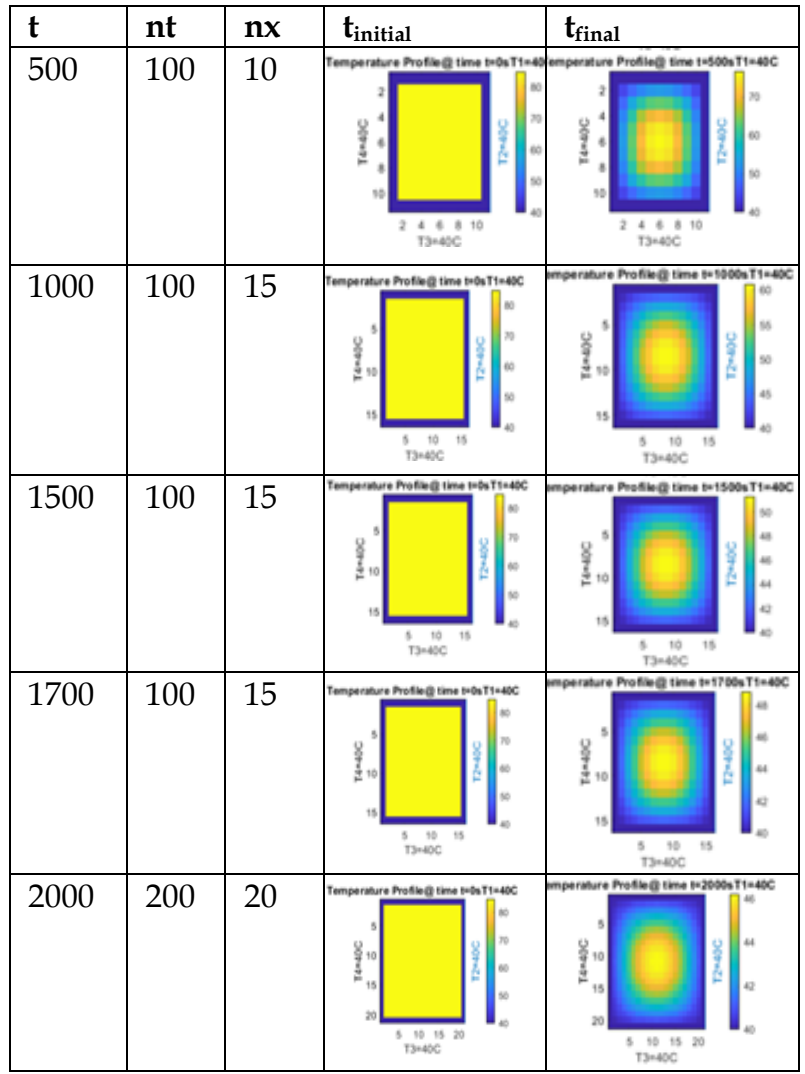

Fig. 14a: Temperature profile showing the effect of varying parameters, nx, nt over varying time t on heat sink behaviour

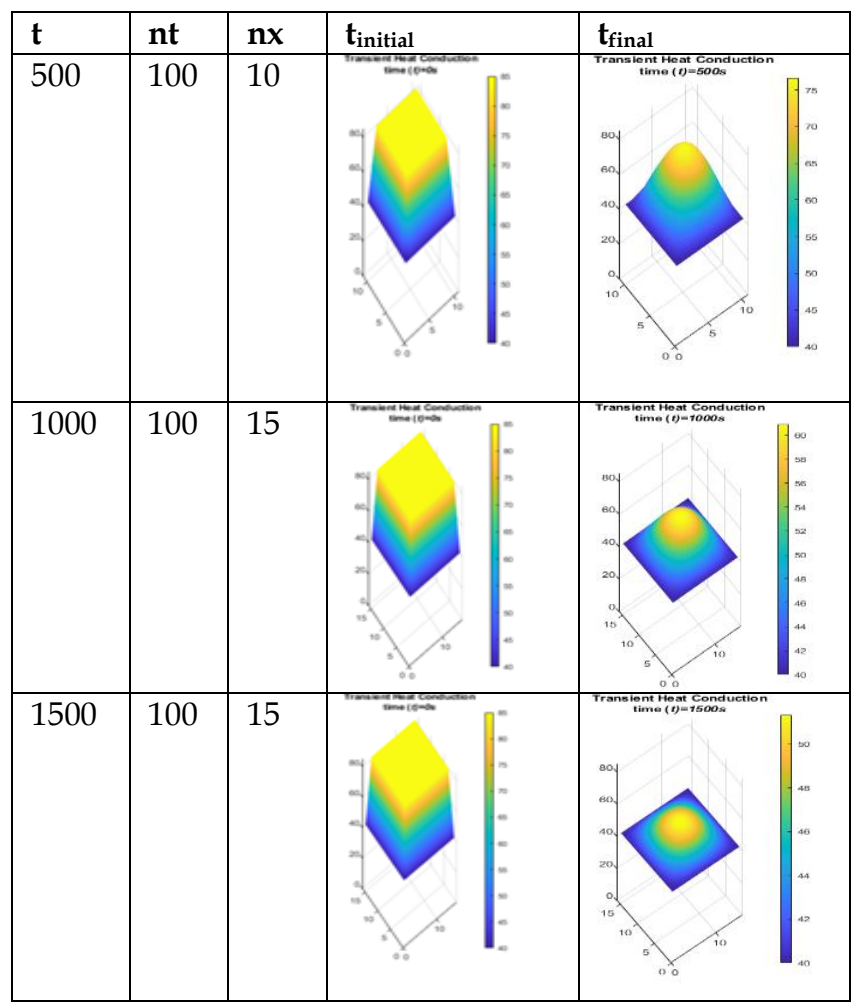




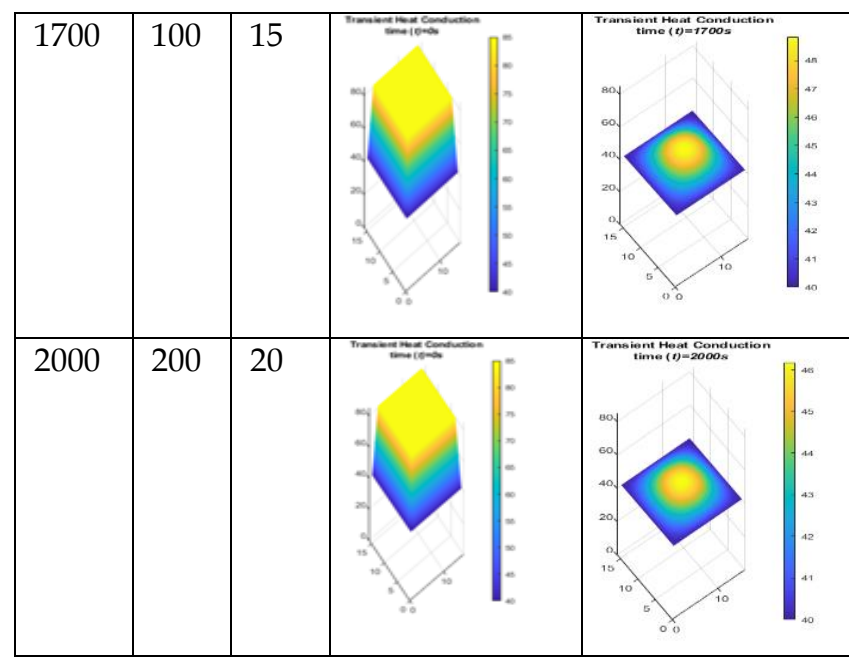

Fig. 14b: Transient heat behaviour showing the effect of varying parameters, $\mathrm{nx}$, nt over varying time $\mathrm{t}$ on heat sink behaviour.

\section{CONCLUSION}

This paper illustrated the accuracy and versatility of the FDTD method by applying it to investigate the effect of LEMP on a transmission line and heat diffusion in a heat sink. Using MATLAB, the effects of varying FDTD parameters on both models were presented. Furthermore, the effect of varying line parameters for the transmission line was presented. Both cases confirmed the ease of application, accuracy and stability of the FDTD method with the key to success being making the right choice of FDTD parameters.

The ability to model lightning-induced voltages provides valuable information from which mitigating actions, such as setting up of LEMP protection measures system, can be implemented with the aim of preventing malfunction or physical damage to line-associated devices and systems. A case in point is in power distribution networks where components and protective/mitigation devices (e.g. surge arresters, shielding wires, and relevant groundings) are specified and implemented during installation to withstand such surges. The ability to accurately model heat diffusion ensures that a close estimate of temperature distribution is obtainable at product design stage of the heat sink and can be confirmed as workable before actual manufacture takes place; thereby saving cost.

The FDM/FDTD has many schemes which include Forward Difference, Backward Difference, Central Difference, Explicit, Implicit and Crank-Nicholson schemes for application with 1-D, 2-D and 3-D solutions. The important step is to select a suitable scheme that will fittingly represent the behaviour of the system. Finally, FDM/FDTD methods can be extended to virtually all the various fields of engineering, technology, sciences and medicine. In fact, its application can be said to be 'limitless' if the computational time and equipment capacity are not considered to be deterring factors; and if the focus is on its ease of implementation. Ease of application implies ease of extension to fields outside engineering and more studies can be carried out on this aspect.

\section{REFERENCES}

Becker, T. W. and Kaus, B. J. P. (2014). Numerical Modeling of Earth Systems. An introduction to computational methods with focus on solid Earth applications of continuum mechanics. 222.

Chakraverty, S., Mahato, N. R., Karunaka, P. and Rao, T. D. (2019). Finite Difference Method. ResearchGate, DOI: 10.1002/9781119423461.ch5

Fauset, L. V. (1999). Applied Numerical Analysis using MATLAB. Prentice Hall.

Gerald, W. (2011). Finite Difference Approximations to the Heat Equation. Portland State University, Portland, Oregon.

Ghazizadeh, H.R., Maerefat, M., and Azimi, A. (2010). Explicit and implicit finite difference schemes for fractional Cattaneo equation. Journal of Computational Physics, (229 (19)), 7042-7057.

Hancock, M. J. (2006). The1-D Heat Equation 18.303 Linear Partial Differential Equations,1-5. Retrieved from https://ocw.mit.edu/courses/mathematics/18-303-linear-partialdifferential-equations-fall-2006/lecture-notes/heateqni.pdf

Kreith, F., Manglik R. M.and Bohn, M. S. (2011). Principles of Heat Transfer, Seventh Edition. Global Engineering, USA.

Kumar, S. (2014). Finite Difference Method: A Brief Study. Available at SSRN: http://dx.doi.org/10.2139/ssrn.2395968

Laughton, M. A. and Warne, D. F., (2003). Electrical Engineer's Reference Book. Newnes $16^{\text {th }}$ Edition; ISBN-10: 0750646376.

Nucci, C. A., Borghetti, A., Paolone, M., Boselli, P., Bernardi, M., Malgarotti, S., Mastandrea, I. and Rachidi, F. (2004). LightningInduced Voltages on Overhead Distribution Lines: Theoretical and Experimental Investigation of related Problems and their Impact on Power Quality, Proceedings of International Council of Large Electric Systems (CIGRE) Symposium. https://e-cigre.org

Offshore Direct Metals, (2020). The Best Aluminum Alloys for Extruded Heat Sinks, https://www.odmetals.com/blog/the-best-aluminumalloys-for-extruded-heat-sinks

Ohnishi, R., Wu, D., Yamaguchi, T. and Ohnuki, S. (2019). Numerical Accuracy of Finite-Difference Methods. International Symposium on Antennas and Propagation, (7), pp. 7-8.

Paolone, M., Rachidi, F., Borghetti, A., Nucci, C. A., Rubinstein, M., Rakov, V. A. and Uman, M. A. (2009). Lightning Electromagnetic Field Coupling to Overhead Lines: Theory, Numerical Simulations, and Experimental Validation, IEEE Transactions on Electromagnetic Compatibility, Vol. 51, No. 3, 532-547.

Pejtamalli, X. and Cipo, P. (2015). Time Domain Finite Difference Numerical Method of Analysis of Direct Lightning Electromagnetic Pulse at Ground Horizontal Wire, European Scientific Journal vol. 2

Pozar, P. M. (2005). Microwave Engineering (Third Edition). John Wiley

Sam R. (2020). 2D Heat Conduction Cart Coordinates Transient FTCS Convection BCs. Retrieved from https://engineeringstream.com/Power_Point_Links/2D_HeatConduction_Cart_Coordina tes_Transient_FTCS - Convection BCs.html

Selvadurai, A. P. S. and Selvadurai, A. P. S. (2000). The diffusion equation. Partial Differential Equations in Mechanics 1, 235-368.

Smith, G. D. (1985). Numerical Solution of Partial Differential Equations: Finite Difference Methods. Oxford University Press.

Smy, T. J., Stewart, S. A. and Gupta, S. (2018). Implicit and Explicit FDTD Methods for Modelling EM Metasurfaces, International Applied Computational Electromagnetics Society (ACES) Symposium, pp. 1-2,

Sjodin, B. (2016). What's the Difference Between FEM, FDM and FVM?, Machine Design, https://www.machinedesign.com

Tsegaye, S. and Purnachandra, R. K. (2016). Application of Some Finite Difference Schemes for Solving One Dimensional Diffusion Equation, American Scientific Research Journal for Engineering, Technology, and Sciences (ASRJETS), 26 (3), 140-154.

Wai, K. K. S. and Tint, S. S. (2019). Finite Difference Methods for Two Dimensional Heat Equation, Iconic Research and Engineering (IRE) Journals, 3(2), 134-138

Wikimedia. (2013). Electromagnetic Pulse. Retrieved November 17, 2019, from https://en.wikipedia.org/wiki/Electromagnetic_Pulse

Yee, K. (1966). Numerical solution of initial boundary value problems involving maxwell's equations in isotropic media. IEEE Transactions on Antennas and Propagation, 14 (3), 302-307

Zhou. P. (1993). Finite Difference Method in numerical analysis of Electromagnetic Fields, Electric Energy Systems and Engineering Series. Springer, Berlin. https://doi.org/10.1007/978-3-642-50319-1 